\title{
Successful transmission probability of cognitive device-to-device communications underlaying cellular networks in the presence of hardware impairments
}

\author{
Hoang-Sy Nguyen ${ }^{1 *}$ (D), Thanh-Sang Nguyen ${ }^{2,3}$ and Miroslav Voznak ${ }^{1,3}$
}

\begin{abstract}
This paper studies the cognitive wireless powered device-to-device (D2D) communication underlaying a cellular network, where there are two types of communications, including peer-to-peer (P2P) communication and a multi-hop D2D communication, which are assumed to be affected by hardware impairments (HWIs). We investigate the one-hop P2P communication and the multi-hop D2D communication, where the relay node helps other two user equipments (UEs) exchange information with a two-time-slot physical-layer network coding scheme. To examine the system performance, we derive closed-form expressions for the average energy efficiency (EE) and spectral efficiency (SE). Besides that, we also obtain the successful transmission probability (STP) for the two considered communications, and the optimal values of time switching (TS) and power splitting (PS) ratios are achieved with the help of a genetic algorithm (GA)-based optimization algorithm in our proposed energy harvesting (EH) protocol so-called Hybrid TS-PS (HTPS) relaying protocol. Comparisons between amplify-and-forward (AF) and decode-and-forward (DF) transmission schemes are provided. The simulation results give evidence that the STP is significantly improved regardless of the presence of HWIs thanks to the derived parameters, i.e., average EE, SE, and the optimal TS and PS ratios.
\end{abstract}

Keywords: Device-and-device, Peer-to-peer, Hardware impairments, Energy harvesting, Power splitting, Time switching, Successful transmission probability, Energy efficiency, Spectral efficiency

\section{Introduction}

It is clear that in wireless communications, energy efficiency (EE) and spectral efficiency (SE) are among the two most important design metrics to cope with environmental problems and the requirements for better wireless communication service. In recent years, simultaneous information and power transfer (SWIPT) has become a promising technology, which allows a wireless equipment to perform energy harvesting $(\mathrm{EH})$ and information processing at the same time by using the ambient radio frequency signals [1-6]. In particular, the work in [7] focused on the analysis of EE in multiple relay orthogonal frequency-division multiplexing (OFDM) systems,

*Correspondence: nguyenhoangsy@tdt.edu.vn

${ }^{1}$ Wireless Communications Research Group, Faculty of Electrical \& Electronics Engineering, Ton Duc Thang University, Ho Chi Minh City, Vietnam

Full list of author information is available at the end of the article in which the information transmission (IT) is assisted by the deployment of a decode-and-forward (DF) relay. In [8], the authors evaluated the successful transmission probability (STP) of cognitive relaying ad hoc networks, where primary users are supported by secondary users in transmitting data packets. Physical-layer network coding (PNC) schemes for two-way relay channels were studied comprehensively, where the transmission mode was studied over two, three, or four time slots [9]. In [10], the SWIPT for the non-regenerative multiple-input multipleoutput OFDM relaying system was studied, where two protocols, including time switching-based relaying (TSR) and power splitting-based relaying (PSR) were deployed at relay nodes. Besides that, the work in [10] solved two optimization problems to optimize the information rate. In [11], a point-to-point wireless link over the flatfading channel was investigated, in which the receiver is 
equipped with power supplies, so it needs energy harvested from signals transferred by transmitter. In [12], the group cooperation was investigated in a wireless $\mathrm{EH}$ network, in which it witnessed the communication of two groups with the help of wireless power transfer and time sharing to satisfy IT. However, in order to significantly enhance the use of spectrum, cognitive radio (CR) is considered as a prime candidate by enabling spectrum sharing [13]. Hence, the combination of cognitive radio networks (CRNs) and EH brings better energy and spectrum.

There have been a number of works on regarding CRNs, where $\mathrm{EH}$ were mentioned in [14-17]. In [14], a dualhop multi-user underlay CRN was examined by using DF scheme and opportunistic scheduling at the destination nodes, where co-channel interference signals were carefully considered for the system design. A multi-antenna cognitive DF was investigated in terms of outage probability, in which the co-existence of secondary source and relay with the primary user (PU) can be maintained in case interference signals affecting PU are below a certain threshold [15]. Besides that, [16] focused on optimal resource allocation policies for CRNs under the spectrum leasing model and putting forward cooperative schemes, in which the time-slot is shared between primary users and secondary users for cooperation. In [17], a hybrid CRN with overlay and underlay models was considered, where a primary link gives the secondary users fractions of its transmission time for cooperation under outage constraints of both primary and secondary networks.

These days, due to the growing demand for better SE and data rates, standards like the 3rd Generation Partnership Project (3GPP) and Long-Term Evolution (LTE) were adopted. Thanks to the further development of LTE-Advanced, promising technologies have been comprehensively investigated, i.e., local area optimization [18]. Furthermore, device-to-device (D2D) communication was proposed as a potential technology in 3GPP Release 12 [19]. In [20], two resource allocating protocols were proposed, and to prove the performance degradation, the system efficiencies were examined in case the unknown channel quality problem was not taken into consideration. In [20, 21], the authors drastically reduced the total amount of power consumed by putting mobile devices (MDs) together in multi-hop D2D networks, while the minimum rate is satisfied to supply each MD. Besides that, the work in [22] tried to enhance throughput in D2Daided underlaying cellular networks, where D2D devices can operate in FD mode to transmit and receive information concurrently with a single-frequency band.

In addition, the distortions stemming from both transmitters and receivers cause hardware impairments (HWIs). In [23], a model under the impact of HWIs at base stations equipped with arrays of large antennas
(BSs) and MDs. Unlike ideal hardware conditions, the authors proved that finite ceilings on the channel estimation accuracy and on the downlink/uplink capacity at each UE are caused by HWIs. Similar to [21], the work in [24] examined the massive MIMO FD relaying network, in which the direct communication of D2D pairs is assisted by a FD relay with enormous arrays of antennas, a low complexity hardware impairments aware (HIA) transceiver scheme was proposed to cope with the distortion noises. In [25], the outage performance of multi-relay decode-and-forward (DF) cooperative networks was considered under the impact of energy constraints and HWIs, in which to address energy constraints at relay nodes, radio-frequency (RF) EH technique was used.

Motivated from our previous works [26-28], where we mainly focused on the impact of HWIs on particular nodes, i.e., relays. In particular, in [26] and [27], the impact of HWIs was considered in AF using TSR protocol and PSR protocol in EH relaying networks, while DF transmission scheme was investigated in [28] using TSR protocol. Regarding multi-hop D2D communication and P2P communication, there have been only a few investigations carried out on the impact of HWIs. Therefore, in order to expand our works on HWIs, we are going to quantify the energy consumption by giving closed-form expressions for the average EE and average SE in case all nodes in the network suffer from HWIs. In addition, we also provide comparisons between AF and DF transmission schemes in the multi-hop D2D communication and the direct P2P communication, and the optimization problem related to TS and PS ratios are also solved in our proposed protocol Hybrid TS-PS (HTPS) relaying protocol. Most importantly, our main goal is to optimize the STP.

The rest of the paper is organized as follows: The system model is discussed in Section 2. Meanwhile, in Section 3, we analyze the system performance in details by examining the impact of HWIs in both communication types and derive closed-form expressions for the STP, average $\mathrm{EE}$, average SE in AF and DF schemes for two communication types, and the achievement of optimal TS and PS ratios is also presented. Section 4 provides numerical and simulation results with detailed analysis and comparisons. Finally, we draw a conclusion for the paper in Section 5.

\section{System model}

As illustrated in Fig. 1, the cognitive device-to-device (D2D) communication underlaying a cellular network is presented, in which we consider in two different modes, including mode A and mode B for simplicity. In particular, in mode A, a multi-hop D2D communication is examined utilizing the two-time-slot physical-layer network coding scheme [9], where two primary user equipments (i.e., UE1 and UE2) establish communication with the help of 


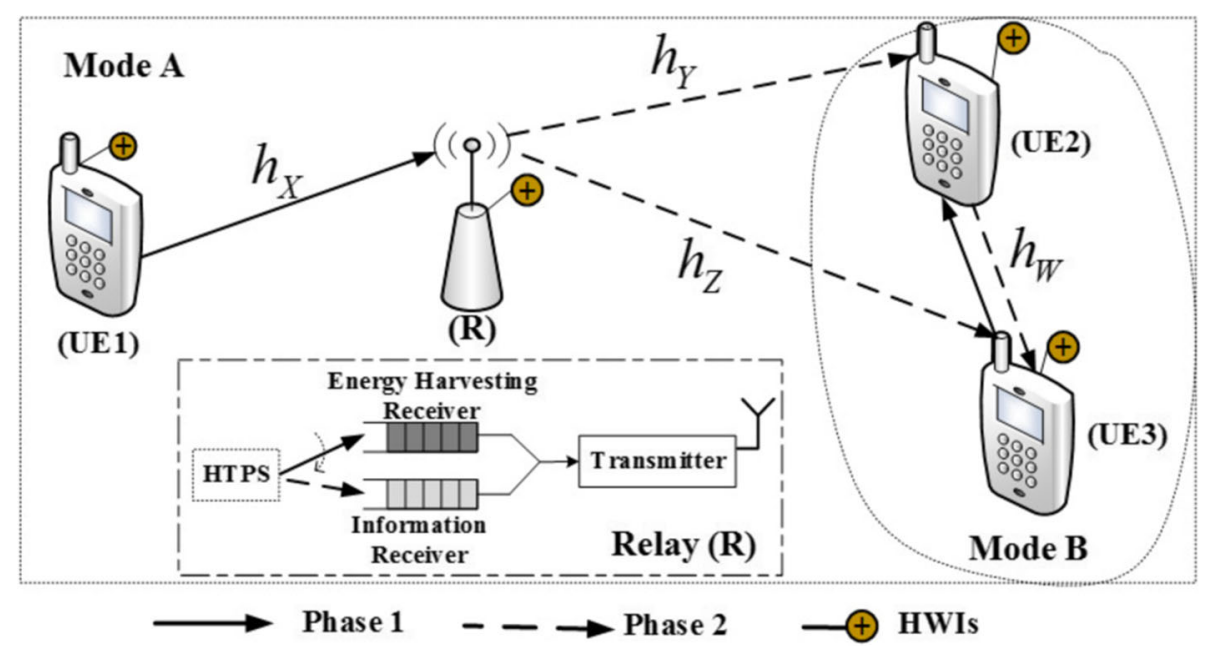

Fig. 1 System model

a secondary EH relay $(\mathrm{R})$ due to the far distance between UE1 and UE2, while mode B describes the one-hop peerto-peer (P2P) wireless communication between UE2 and UE3, where UE2 and UE3 exchange information directly with each other. It is noted that $\mathrm{R}$ has ability to transfer information intended to UE3, so both primary and secondary information can be carried by $\mathrm{R}$ at the same time. All UEs operate in half-duplex (HD) mode and are equipped with a single antenna.

Let us start with mode A, the whole operation includes two phases. In particular, in phase 1, UE1 transmits the information to R. After harvesting energy from part of the received signal transmitted from UE1, R exploits the harvested energy to broadcast resulting signals as well as the information intended to UE2 and UE3 in phase 2. It is worth noting that UE3 can receive signals to conduct interference cancelation in phase 2. Meanwhile, in case of mode B, UE2 and UE3 will exchange information with each other via the P2P link within two time slots.

We assume that both the maximum threshold of the transmission power and the circuit power for UEs are the same, which are denoted as $E_{D i}$ and $E_{C i}$, respectively, with $i=1,2,3$, we denote $E_{D i}=E_{D}$ and $E_{C i}=E_{C}$ for simplicity. In case $\mathrm{R}$ suffers from a lack of energy due to limited battery life, it needs to depend on external energy charging to keep the network active. Note that the transmit power of $\mathrm{R}$ is denoted as $E_{R}$. Besides that, $n_{0}$ is the additive white Gaussian noise (AWGN) with mean power, $\Omega_{0}$.

In terms of system channels, the channel gains are denoted as $h_{X}, h_{Y}$, and $h_{Z}$ for the link from UE1 to R, and $\mathrm{R}$ to UE2, UE3, respectively, while the corresponding channel gain for the P2P communication between UE2 and UE3 is $h_{W}$. Furthermore, $r_{k}$ is the distances for the aforementioned links, where $k \in\{X, Y, Z, W\}$.
In addition, we denote $P L_{k}$ as the path loss model which is defined as $P L_{k}=1 / \phi r_{k}^{m}$ with $m$ being the path-loss exponent, and $\phi$ is the path-loss constant [29]. We model all channel gains between two nodes as Rayleigh fading channels with free space propagation path loss. Specifically, for the channel gain coefficient, we have $\left|h_{k}\right|^{2}=$ $|k|^{2} P L_{k}$, in which $k$ is the complex Gaussian distributed random variable $(\mathrm{RV})$ to model fading phenomena with zero mean and variances, $\Omega_{k} \sim C N(0,1)$.

Regarding the protocol deployed in the paper, a hybrid TS and PS receiver architecture is proposed so-called Hybrid TS-PS (HTPS) relaying protocol which results in a generalized version of PSR protocol and TSR protocol [4], in which $\alpha$ and $\beta$ are denoted as TS and PS fraction, respectively. In the block time $T$, the portion, $\alpha_{1} T$ is used for $E H$ at $\mathrm{R}$ with $E_{D}$. Source signals transmitted from UE1 to $\mathrm{R}$ are split into two parts. In particular, during the portion, $\alpha_{2} T, \beta E_{D}$ is used for $\mathrm{EH}$ at $\mathrm{R}$ while a fraction, $(1-\beta) E_{D}$ belongs to IT between UE1 and R. Eventually, the remaining fraction, $\left(T-\alpha_{1} T-\alpha_{2} T\right)$ is used for broadcasting signals from $\mathrm{R}$ to UE2, UE3 with power allocation, $\lambda$ and $(1-\lambda)$, respectively. Note that PSR protocol is a special case of this hybrid TS-PS relaying protocol when $\alpha_{1}=0$ and $\alpha_{2}=0.5$, while TSR protocol is a special case when $\beta=0$ and $\alpha_{2}=0.5 \times\left(1-\alpha_{1}\right)$.

It is worth noting that all nodes suffer from HWIs caused by UEs [30]. The practical transceiver impairments at UEs denoting as $x$ within the $a \rightarrow b$ link, while the HWIs of the receivers compromise the received signal during the reception phase. Each distortion of UE1 can be represented by a different model. We denote $H I_{a}$ and $H I_{b}$ as the aggregate distortions affecting the $a \rightarrow b$ link with zero mean variance $H I_{a} \sim C N\left(0, \kappa_{a}|h|^{2} E_{D}\right), H I_{b} \sim$ $C N\left(0, \kappa_{b}|h|^{2} E_{D}\right)$, respectively, and $\kappa_{a}$ and $\kappa_{b}$ are the levels 
of HWIs at $H I_{a}$ and $H I_{b}$. Therefore, the received signal can be expressed as

$$
\begin{aligned}
y_{b} & =\sqrt{E_{D}} h x+H I_{a}+H I_{b}+n_{0}, \\
& =\sqrt{E_{D}} h x+H I_{a b}+n_{0}
\end{aligned}
$$

where $h$ is the channel gain for the $a \rightarrow b$ link, and an aggregate distortion at the receiver $b$ is denoted as $H I_{a b}$ with aggregate distortion power $H I_{a b} \sim C N\left(0, \kappa|h|^{2} E_{D}\right)$, such that $\kappa \triangleq \kappa_{a}+\kappa_{b}$ is the aggregate impairment level during the information processing phase [31].

Hence, the distortion noise stemming from HWIs at $\mathrm{R}$ is represented by $H I_{1}$ with variance $H I_{1} \sim$ $\mathrm{CN}\left(0, \kappa \mathrm{E}_{\mathrm{D}} \mathrm{PL}_{\mathrm{X}}|\mathrm{X}|^{2}\right)$, while the corresponding distortion noise caused by HWIs at UE2 and UE3 are denoted by $H I_{2}$ and $H I_{3}$ with variance $H I_{2} \sim C N\left(0, \kappa \lambda E_{R}|Y|^{2} P L_{Y}\right)$, $H_{3} \sim C N\left(0, \kappa(1-\lambda) E_{R}|Z|^{2} P L_{Z}\right)$, respectively.

For simplicity, we list important notations used in this paper in Table 1.

\section{Performance analysis under the impact of HWIs} In this section, in terms of analyzing the system performance, we are going to examine the proposed HTPS relaying protocol in mode $\mathrm{A}$, and closed-form expressions for the successful transmission probability (STP), average energy efficiency (EE), and average spectral efficiency (SE) are derived. More importantly, we achieve the optimal values of TS and PS ratios.

\subsection{Energy harvesting and information transmission under the impact of HWls \\ 3.1.1 Mode A: multi-hop cognitive D2D wireless communication}

In this section, we investigate the impact of HWIs based on the architecture of HTPS relaying protocol.

Phase 1 At first, the amount of energy harvested at R can be expressed as

$$
E_{h}=\eta E_{D}|X|^{2} P L_{X}\left(\alpha_{1}+\alpha_{2} \beta\right) T,
$$

where $\eta$ is the energy conversion efficiency and $\eta \in(0,1]$.

Hence, the transmit power of $\mathrm{R}$ can be computed by

$$
E_{R}=\frac{E_{h}}{\left(1-\alpha_{1}-\alpha_{2}\right) T}=\frac{\eta\left(\alpha_{1}+\alpha_{2} \beta\right) E_{D} P L_{X}|X|^{2}}{1-\alpha_{1}-\alpha_{2}} .
$$

The received information at $\mathrm{R}$ can be depicted as

$$
y_{1}=\sqrt{(1-\beta) E_{D}} h_{X x_{1}}+n_{0}+H I_{1},
$$

Table 1 Important notations

\begin{tabular}{ll}
\hline Symbols & Meanings \\
\hline$\eta$ & The energy conversion efficiency \\
$E_{D}$ & The maximum transmission power of UE1, UE2, and \\
$E_{C}$ & UE3. \\
$E_{R}$ & The circuit power of UE1,UE2, and UE3. \\
$|X|^{2}, T_{X}$, The transmit power of $\mathrm{R}$
\end{tabular}

$|X|^{2}, r_{X}$, and $P L_{X} \quad$ The channel power gain, the distance, and the path loss model for the link from UE1 to R, respectively.

$|Y|^{2}, r_{Y}$, and $P L_{Y} \quad$ The channel power gain, the distance, and the path loss model for the link from $\mathrm{R}$ to UE2, respectively.

$|Z|^{2}, r z$, and $P L Z$

The channel power gain, the distance, and the path loss model for the link from R to UE3, respectively.

$|W|^{2}, r_{w}$, and $P L_{W}$

The channel power gain, the distance, and the path loss model for the link from UE2 to UE3, respectively.

$\Omega_{k}$

The exponential parameters corresponding to $|k|^{2}$, $k \in[X, Y, Z, W]$.

$\alpha_{1} T$

$\alpha_{2} T$

Time switching (TS) fraction used for EH from UE1 to $\mathrm{R}$

TS fraction used for IT from EU1 to $R$

$\left(T-\alpha_{1} T-\alpha_{2} T\right) \quad$ TS used for IT from R to UE2 and UE3

$\beta E_{D}$ Power spitting (PS) fraction used for $E H$ at $\mathrm{R}$

$(1-\beta) E_{D}$

$\lambda$ Information transmission (IT) from UE1 to $R$ in PS protocol

The power allocation at $\mathrm{R}$ used to transmit signals to UE2

$(1-\lambda)$

The power allocation at R used to transmit signals to UE3

$\kappa$

$H_{1}$

$\mathrm{HI}_{2}, \mathrm{H}_{3}$, and $\mathrm{HI}_{4}$

$n_{0}$

$F_{x}, f_{x}$

$\operatorname{Pr}\{$.

$K_{n}\{$.

$\mathbb{E}\{\}$

$\gamma_{i}^{A F}, \gamma_{i}^{D F}$

$\Gamma_{D}$
The aggregate level of hardware impairments

The distortion noise caused by HWls in the communication between UE1 and $\mathrm{R}$ with variance, $H_{1} \sim \mathrm{CN}\left(0, \kappa \mathrm{E}_{\mathrm{D}} \mathrm{PLX}|\mathrm{X}|^{2}\right)$

The distortion noise HWIs from $\mathrm{R}$ to UE2, $\mathrm{R}$ to UE3, and between UE2 and UE3 with variance $H_{2} \sim \mathrm{CN}\left(0, \kappa \lambda E_{R} P L Y|Y|^{2}\right), H H_{3} \sim \mathrm{CN}(0, \kappa(1-\lambda)$ $\left.E_{R} P L_{Z}|Z|^{2}\right)$, and $H I_{4} \sim C N\left(0, \kappa E_{D} P L W|W|^{2}\right)$, respectively.

The additive white Gaussian noise (AWGN) at UEs and $\mathrm{R}$ with mean power, $\Omega_{0}$,

The cumulative distribution function (CDF)/the probability density function (PDF) of the RV $x$ is $F_{x}(z)=\operatorname{Pr}(x<z)=1-e^{-\frac{z}{\Omega_{x}}}, f_{x}(z)=\operatorname{Pr}(x<$ $z)=\frac{z}{\Omega_{x}} e^{-\frac{z}{\Omega_{x}}}$, respectively, in which $\Omega_{x}$ is the mean of the exponential RV $x$.

The probability distribution function.

The $n$ order modified Bessel function of the second kind with the last equality

The expectation operator.

The signal-to-noise-plus-distortion ratio (SNDR) at UEi for AF scheme and DF scheme, respectively

The SNDR threshold

where the normalized data signal from UE1 is $x_{1}$, which satisfies $\mathbb{E}\left\{\left|x_{1}\right|^{2}\right\}=1$. 
Due to the wireless broadcast nature, the harvested power at $\mathrm{R}$ is split into two streams, $\lambda E_{R}$ is utilized for forwarding signals to the primary node, UE2 while $(1-\lambda) E_{R}$ is for transferring signals to the secondary node, UE3. It is worth noting that we are going to consider both AF and DF transmission scheme.

Therefore, let us start with DF scheme, where the the transmitted signal at $\mathrm{R}, x_{R}$ can be expressed by

$$
x_{R}=\left(\sqrt{\lambda E_{R}} x_{2}+\sqrt{(1-\lambda) E_{R}} x_{3}\right)
$$

where $x_{2}$ and $x_{3}$ are the unit power of the transmitted information intended for UE2 and UE3, respectively, which satisfies $\mathbb{E}\left\{\left|x_{2}\right|^{2}\right\}=1$, and $\mathbb{E}\left\{\left|x_{3}\right|^{2}\right\}=1$.

Regarding the transmitted signal at R in AF scheme, $x_{R}$ can be expressed as

$$
x_{R}=G \sqrt{\lambda E_{R}} y_{1}+\sqrt{(1-\lambda) E_{R}} x_{3}
$$

where the amplification factor at $\mathrm{R}$ [26] is calculated as

$$
\begin{aligned}
& G=\sqrt{\left((1-\beta) E_{D}|X|^{2} P L_{X}+\Omega_{0}+\kappa E_{D}|X|^{2} P L_{X}\right)^{-1}} \approx \\
& \sqrt{\left((1-\beta+\kappa) E_{D}|X|^{2} P L_{X}\right)^{-1}} \text {. }
\end{aligned}
$$

Remark 1 It is worth noting that choosing the power allocation, $\lambda$ is the main point because it is essential to balance the received information at UE2 and UE3. Hence, in order to maintain the quality of UE2 at an acceptable condition, as $\lambda$ increases, the amount of signal received at UE3 falls. Later on in this paper, we are going to explain this scenario in details in Remark 2.

Phase 2 In terms of the IT process between R and UE2, the corresponding received signal at UE2 can be shown by

$$
y_{2}=h_{Y} x_{R}+n_{0}+H I_{2}
$$

and the received signal at UE3 can be written as

$$
y_{3}=h_{Z} x_{R}+n_{0}+H I_{3} .
$$

\subsubsection{Mode B: $P 2 P$ wireless communication}

In this mode, the P2P communication between UE2 and UE3 is carried out in two time slots. We assume the same signal is received at UE2 and UE3, which can be expressed by

$$
y_{4}=\sqrt{E_{D}} h_{W} x_{4}+n_{0}+H I_{4}
$$

where $x_{4}$ is the transmitted data from UE2 to UE3 and vice versa, which satisfies $\mathbb{E}\left\{\left|x_{4}\right|^{2}\right\}=1$, and the distortion noise caused by HWIs at UE2 and UE3 are denoted as $H I_{4}$, where $\Omega_{4} \sim C N\left(0, \kappa E_{D}|W|^{2} P L_{W}\right)$. Note that $P L_{W}=\left(\phi r_{W}^{m}\right)^{-1}$.

\subsection{The end-to-end signal-to-noise-plus-distortion ratio (SNDR)}

In this part, we investigate the end-to-end signal-tonoise-plus-distortion ratio (SNDR), $\gamma$, where $\gamma=$ $\mathbb{E}\left\{\mid\right.$ signal $\left.\left.\right|^{2}\right\} / \mathbb{E}\left\{\mid\right.$ overall noise $\left.\left.\right|^{2}\right\}$ for AF and DF scheme under the impact of HWIs. Let us start with AF scheme.

\subsubsection{AF case}

By substituting (3), (4) into (6), then we combine with (7), the end-to-end SNDR at UE2 can be expressed when UE2 considers $x_{3}$ as interference and then decodes the primary information, $x_{1}$ as

$$
\gamma_{1}^{A F}=\frac{\tau_{1, a}|X|^{2}|Y|^{2}}{\tau_{1, b}|X|^{2}|Y|^{2}+\tau_{1, c}|Y|^{2}+\tau_{0}}
$$

$\begin{array}{lll}\text { where } \delta_{1}= & \kappa \lambda \\ (1-\lambda)(1-\beta+\kappa), & \tau_{0}= & +\begin{array}{c}\kappa \lambda(1-\beta+\kappa) \\ (1-\beta+\kappa)\left(1-\alpha_{1}-\alpha_{2}\right)\end{array}\end{array}$ and $(1-\lambda)(1-\beta+\kappa), \quad \tau_{0}=\frac{(1-\beta+\kappa)\left(1-\alpha_{1}-\alpha_{2}\right)}{\eta\left(\alpha_{1}+\alpha_{2} \beta\right)}$, and $\left\{\begin{array}{l}\tau_{1, a}=\frac{E_{D}}{\Omega_{0}}(1-\beta) \lambda P L_{X} P L_{Y} \\ \tau_{1, b}=\frac{E_{D}}{\Omega_{0}} \delta_{1} P L_{X} P L_{Y} \\ \tau_{1, c}=\lambda P L_{Y} .\end{array}\right.$

Likewise, replacing (3), (4), and (6) into (8), we derive the end-to-end SNDR at UE3 in case UE3 treats $x_{1}$ as interference and then decodes the secondary information, $x_{3}$ as follows

$$
\gamma_{2}^{A F}=\frac{\tau_{2, a}|X|^{2}|Z|^{2}}{\tau_{2, b}|X|^{2}|Z|^{2}+\tau_{2, c}|Z|^{2}+\tau_{0}},
$$

where $\delta_{2}=\kappa \lambda+\kappa(1-\lambda)(1-\beta+\kappa)+\lambda(1-\beta-\kappa)+\kappa$, $\tau_{0}$ is mentioned in the above expression, and $\left\{\begin{array}{l}\tau_{2, a}=\frac{E_{D}}{\Omega_{0}}(1-\beta)(1-\lambda) P L_{X} P L_{Z} \\ \tau_{2, b}=\frac{E_{D}}{\Omega_{0}} \delta_{2} L_{X} P L_{Z} \\ \tau_{2, c}=\lambda P L_{Z} .\end{array}\right.$

As a result, the data rate achieved at UE2 and UE3 can be given by

$$
r_{i}^{A F} \underset{i \in\{1,2\}}{=} \frac{B}{2} \log _{2}\left(1+\gamma_{i}^{A F}\right)
$$

where $B$ denotes the channel bandwidth.

Remark 2 In order to clarify and expand Remark 1, since the end-to-end SNDR at UE2 is expressed to satisfy the quality of service. In particular, when secondary signals are treated as interference, it is straightforward to obtain from (10) that if $E_{D} \rightarrow \infty$ in case all parameters are fixed, $\lim _{E_{D \rightarrow \infty}} \gamma_{1}^{A F}=\lambda /(1-\lambda)$. In contrast, since the end-to-end SNDR at UE3 (11) is computed, primary signals are considered as interference, where if $E_{D} \rightarrow \infty$, $\lim _{E_{D \rightarrow \infty}} \gamma_{2}^{A F}=(1-\lambda) / \lambda$. These phenomena are going to be examined by simulations. 


\subsubsection{DF case}

Similarly, regarding DF scheme, the end-to-end SNDR at both UE2 and UE3 can be written as

$$
\gamma_{i}^{D F} \underset{i \in\{1,2\}}{=} \min \left\{\gamma_{i, a}^{D F}, \gamma_{i, b}^{D F}\right\}
$$

where the instantaneous SNDR at R, UE2, and UE3 can be computed, respectively, based on (4), (7), and (8) as follows $\left\{\begin{array}{l}\gamma_{1, a}^{D F}=\gamma_{2, a}^{D F}=\frac{(1-\beta) \frac{E_{D}}{\Omega_{0}} P L_{X}|X|^{2}}{1+\kappa \frac{E_{D}}{\Omega_{0}} P L_{X}|X|^{2}} \\ \gamma_{1, b}^{D F}=\frac{\psi_{1, a}|X|^{2}|Y|^{2}}{\psi_{1, b}|X|^{2}|Y|^{2}+\psi_{0}} \\ \gamma_{2, b}^{D F}=\frac{\psi_{2, a}|X|^{2}|Z|^{2}}{\psi_{2, b}|X|^{2}|Z|^{2}+\psi_{0}}\end{array}\right.$, and $\psi_{0}=\frac{\left(1-\alpha_{1}-\alpha_{2}\right)}{\eta\left(\alpha_{1}+\alpha_{2} \beta\right)}, \quad \psi_{1, a}=\frac{E_{D}}{\Omega_{0}} \lambda P L_{X} P L_{Y}, \psi_{2, a}=$ $\frac{E_{D}}{\Omega_{0}}(1-\lambda) P L_{X} P L_{Z}, \quad \psi_{1, b}=\frac{E_{D}}{\Omega_{0}}(\kappa \lambda+1-\lambda) P L_{X} P L_{Y}$, $\psi_{2, b}=\frac{E_{D}}{\Omega_{0}}(\kappa(1-\lambda)+\lambda) P L_{X} P L_{Z}$.

Therefore, the transmission rate for DF protocol is given by

$$
r_{i}^{D F} \underset{i \in\{1,2\}}{=} \frac{B}{2} \log _{2}\left(1+\gamma_{i}^{D F}\right) .
$$

\subsubsection{Peer-to-peer case}

In this part, we are going to study the SNDR under the impact of HWIs in P2P communication as follows

$$
\gamma_{3}^{P P}=\frac{\frac{E_{D}}{\Omega_{0}} P L_{W}|W|^{2}}{1+\kappa \frac{E_{D}}{\Omega_{0}} P L_{W}|W|^{2}}
$$

\subsection{Analysis on successful transmission probability, average energy efficiency, and average spectral efficiency}

In this section, we are going to study on the successful transmission probability (STP), the average energy efficiency (EE), and the average spectral efficiency (SE) in the presence HWIs based on SNDR. Besides that, comparisons between AF and DF schemes are evaluated in multihop D2D communication and P2P communication, and we also provide closed-form expressions for each factor. Most importantly, the optimization problem regarding TS and $\mathrm{PS}$ ratios are solved.

\subsubsection{Successful transmission probability}

It is noted that the STP based on SNDR represents the probability that a receiver could receive successfully packets in the up-link from the transmitter in a time slot. Considering the STP in the one-hop P2P communication, packets are successfully received if the SNDR is greater than the SNDR threshold, $\Gamma_{D}, \operatorname{Pr}\left(\gamma \geq \Gamma_{D}\right)$. Otherwise, UE2 and UE3 will receive a negative feedback and the packets are still put first in the queue for being retransmitted. Therefore, the STP in P2P communication can be derived as follows

$$
\begin{aligned}
\operatorname{Pr}\left(\gamma_{3}^{P P} \geq \Gamma_{D}\right) & =\operatorname{Pr}\left(\frac{\frac{E_{D}}{\Omega_{0}} P L_{W}|W|^{2}}{1+\kappa \frac{E_{D}}{\Omega_{0}} P L_{W}|W|^{2}} \geq \Gamma_{D}\right) \\
& =1-\operatorname{Pr}\left(\frac{\frac{E_{D}}{\Omega_{0}} P L_{W}|W|^{2}}{1+\kappa \frac{E_{D}}{\Omega_{0}} P L_{W}|W|^{2}}<\Gamma_{D}\right) \\
& =e^{-\frac{\Omega_{0} \Gamma_{D}}{\Omega_{W} E_{D} P L_{W}\left(1-\kappa \Gamma_{D}\right)}}
\end{aligned}
$$

In terms of multi-hop D2D communication in the presence of HWIs with the help of the secondary relay, we are going to obtain the STP which undergoes large-scale path loss and small-scale Rayleigh fading at UE2 and UE3 in AF and DF scheme in Propositions 1 and 2, respectively.

Proposition 1 Therefore, the STP at UE2 and UE3 in AF scheme in case $\Gamma_{D} \geq \tau_{i, a} / \tau_{i, b}$ is given by

$$
\operatorname{Pr}\left(\gamma_{i}^{A F} \geq \Gamma_{D}\right)_{i \in\{1,2\}}^{=} 1
$$

Otherwise, in case $\Gamma_{D}<\tau_{i, a} / \tau_{i, b}$, we derive the STP at UE2 and UE3 as

$$
\operatorname{Pr}\left(\gamma_{i}^{A F} \geq \Gamma_{D}\right) \underset{i \in\{1,2\}}{=} 2 e^{-\omega_{i}^{A F}} \sqrt{\vartheta_{i}^{A F}} K_{1}\left(2 \sqrt{\vartheta_{i}^{A F}}\right),
$$

where $\omega_{i}^{A F}=\frac{\Gamma_{D} \tau_{i, c}}{\Omega_{X}\left(\tau_{i, a}-\Gamma_{D} \tau_{i, b}\right)}$, and $\vartheta_{i}^{A F}=\frac{\Gamma_{D} \tau_{0}}{\Omega_{X} \Omega_{Y}\left(\tau_{i, a}-\Gamma_{D} \tau_{i, b}\right)}$.

Proof Let us start with AF scheme, where the general SNDR for both UE2 and UE3 can be written as

$$
\gamma_{i}^{A F}=\frac{a X Y}{b X Y+c Y+d},
$$

where $a, b, c$, and $d$ are constant values, and the exponential random variables, i.e., $X$ and $Y$ are independent with means, $\Omega_{X}$ and $\Omega_{Y}$, respectively.

Based on (19), the CDF of SNDR can be written as

$$
\begin{aligned}
& \operatorname{Pr}\left(\gamma_{i}^{A F}<\Gamma_{D}\right) \\
& =\operatorname{Pr}\left(X<\frac{\Gamma_{D}(c Y+d)}{Y\left(a-\Gamma_{D} b\right)}\right) \\
& =\frac{1}{\Omega_{Y}} \int_{y=0}^{\infty}\left(1-e^{-\frac{\Gamma_{D}(c y+d)}{\Omega_{X} y\left(a-\Gamma_{D} b\right)}}\right) e^{-\frac{y}{\Omega_{Y}}} d y \\
& =1-2 e^{-\frac{\Gamma_{D}}{\Omega_{X}\left(a-\Gamma_{D} b\right)}} \sqrt{\frac{\Gamma_{D} d}{\Omega_{X} \Omega_{Y}\left(a-\Gamma_{D} b\right)}} K_{1}\left(2 \sqrt{\frac{\Gamma_{D} d}{\Omega_{X} \Omega_{Y}\left(a-\Gamma_{D} b\right)}}\right)
\end{aligned}
$$

where we take advantage of the formula [[32], 3.324.1], under the condition, $\Gamma_{D}<\tau_{i, a} / \tau_{i, b}$. In contrast, if $\Gamma_{D} \geq$ $\tau_{i, a} / \tau_{i, b}, \operatorname{Pr}\left(X<\frac{\Gamma_{D}(c Y+d)}{Y\left(a-\Gamma_{D} b\right)}\right)=1$, due to the fact that the probability will be greater than negative values and equal to 1 . 
To this point, the STP at UE2 can be expressed as

$$
\begin{aligned}
\operatorname{Pr}\left(\gamma_{i}^{A F} \geq \Gamma_{D}\right)= & 2 e^{-\frac{\Gamma_{D}}{\Omega_{X}\left(a-\Gamma_{D} b\right)} \sqrt{\frac{\Gamma_{D} d}{\Omega_{X} \Omega_{Y}\left(a-\Gamma_{D} b\right)}}} . \\
& \times K_{1}\left(2 \sqrt{\frac{\Gamma_{D} d}{\Omega_{X} \Omega_{Y}\left(a-\Gamma_{D} b\right)}}\right)
\end{aligned}
$$

This ends the proof for Proposition 1.

Proposition 2 Similar to AF scheme, the STP in case of $D F$ at UE2 and UE3 is computed by

$$
\operatorname{Pr}\left(\gamma_{i}^{D F} \geq \Gamma_{D}\right) \underset{i \in\{1,2\}}{=} 2 e^{-\omega_{i}^{D F}} \sqrt{\vartheta_{i}^{D F}} K_{1}\left(2 \sqrt{\vartheta_{i}^{D F}}\right),
$$

$$
\begin{aligned}
& \text { where } \omega_{i}^{D F} \\
& \frac{\Gamma_{D} \psi_{0}}{\Omega_{X} \Omega_{Z}\left(\psi_{i, a}-\Gamma_{D} \psi_{i, b}\right)}
\end{aligned}
$$

Proof First, we use (4), so the CDF of the instantaneous SNDR in the first hop from UE1 to $R$ is given by

$$
\begin{gathered}
\operatorname{Pr}\left(\gamma_{i a}^{D F}<\Gamma_{D}\right)=\operatorname{Pr}\left(\frac{(1-\beta) \frac{E_{D}}{\Omega_{0}} P L_{X}|X|^{2}}{1+\kappa \frac{E_{D}}{\Omega_{0}} P L_{X}|X|^{2}}<\Gamma_{D}\right) . \\
=1-e^{-\frac{\Gamma_{D} \Omega_{0}}{\Omega_{X} E_{D} P L_{X}\left((1-\beta)-\kappa \Gamma_{D}\right)}}
\end{gathered}
$$

Following that, based on (23), the STP in the first hop from UE1 to R can be written by

$$
\operatorname{Pr}\left(\gamma_{i a}^{D F} \geq \Gamma_{D}\right)=e^{-\frac{\Gamma_{D} \Omega_{0}}{\Omega_{X} E_{D} P L_{X}\left((1-\beta)-\Gamma_{\left.D^{\kappa}\right)}\right.}} .
$$

If $\Gamma_{D}<\psi_{i, a} / \psi_{i, b}$, the STP of the instantaneous SNDR from $\mathrm{R}$ to UE2, UE3 in the second hop can be computed based on (7), (8) as

$$
\begin{aligned}
\operatorname{Pr}\left(\gamma_{i b}^{D F} \geq \Gamma_{D}\right)= & \operatorname{Pr}\left(\frac{\psi_{i, a} X Y}{\psi_{i, b} X Y+\psi_{0}} \geq \Gamma_{D}\right) \\
= & 1-\operatorname{Pr}\left(\frac{\psi_{i, a} X Y}{\psi_{i, b} X Y+\psi_{0}}<\Gamma_{D}\right) \\
= & \frac{1}{\Omega_{Y}} \int_{y=0}^{\infty}\left(e^{-\frac{1}{y}\left(\frac{\Gamma_{D} \psi_{0}}{\Omega_{X}\left(\psi_{i, a}-\Gamma_{D} \psi_{i, b}\right)}\right)}\right) e^{-\frac{y}{\Omega_{Y}}} d y \\
= & \left.2 \sqrt{\frac{\Gamma_{D} \psi_{0}}{\Omega_{X} \Omega_{Y}\left(\psi_{i, a}-\Gamma_{D} \psi_{i, b}\right)}}\right) \\
& \times K_{1}\left(2 \sqrt{\frac{\Gamma_{D} \psi_{0}}{\Omega_{X} \Omega_{Y}\left(\psi_{i, a}-\Gamma_{D} \psi_{i, b}\right)}}\right)
\end{aligned}
$$

Otherwise, if $\Gamma_{D} \geq \psi_{i, a} / \psi_{i, b}$, then $\operatorname{Pr}\left(\frac{\psi_{i, a} X Y}{\psi_{i, b} X Y+\psi_{0}} \geq \Gamma_{D}\right)=0$.

Eventually, by denoting the end-to-end SNDR at UE2, UE3 in DF scheme as $\gamma_{i}^{D F}=\min \left(\gamma_{i a}^{D F}, \gamma_{i b}^{D F}\right)$ with $i \in$ $\{1,2\}$, the STP of $\gamma_{i}^{D F}$ can be given by

$$
\operatorname{Pr}\left(\gamma_{i}^{D F} \geq \Gamma_{D}\right)=\operatorname{Pr}\left(\gamma_{i a}^{D F} \geq \Gamma_{D}\right) \times \operatorname{Pr}\left(\gamma_{i b}^{D F} \geq \Gamma_{D}\right) .
$$

Replacing (24), (25) into (26), we can easily end the proof for Proposition 2.

Remark 3 Finding the joint optimal values of TS and PS for EH that help maximize the STP is essential. It may be challenging to evaluate the joint optimal values of TS and PS ratios in terms of the STP with Bessel function for the given system parameters, including maximum transmission power, distance, power allocation and HWIs level, etc. Due to the non-convex optimization problem which cannot be solved easily, we are going to analyze in details how we deploy a genetic algorithm (GA)-based optimization algorithm in Section 3.4.

\subsubsection{Average energy efficiency and average spectral efficiency}

In this part, we are going to further obtain average EE and average SE. Note that EE is defined as the average transmission rate under unit-energy consumption. In order to achieve energy-efficient communication, both the transmission power and the circuit power can be examined [5].

Proposition 3 We derive the expression for the average $E E$ and average $S E$, respectively, in AF scheme as

$$
\begin{aligned}
e e_{i}^{A F} & =\mathbb{E}\left\{\frac{B \log _{2}\left(1+\gamma_{i}^{A F}\right)}{2 E_{\text {sum }}}\right\} \\
& =\frac{B}{2 E_{\text {sum }}} \int_{x=0}^{\tau_{i, a} / \tau_{i, b}}\left(M_{i}^{A F}+N_{i}^{A F}\right) \log _{2}(1+x) d x
\end{aligned}
$$

and

$$
\begin{aligned}
s e_{i}^{A F} & =\mathbb{E}\left\{\frac{B \log _{2}\left(1+\gamma_{i}^{A F}\right)}{2 B}\right\} \\
& =\frac{1}{2} \int_{x=0}^{\tau_{i, a} / \tau_{i, b}}\left(M_{i}^{A F}+N_{i}^{A F}\right) \log _{2}(1+x) d x
\end{aligned}
$$

where $M_{i}^{A F}=\frac{2 \tau_{i, a} e^{-\omega_{i}^{A F} \vartheta_{i}^{A F} K_{0}\left(2 \sqrt{\vartheta_{i}^{A F}}\right)}}{x\left(\tau_{i, a}-x \tau_{i, b}\right)}, N_{i}^{A F}=$ $\frac{2 \tau_{i, a} \omega_{i}^{A F} e^{-\omega_{i}^{A F}} \sqrt{\vartheta_{i}^{A F}} K_{1}\left(2 \sqrt{\vartheta_{i}^{A F}}\right)}{x\left(\tau_{i, a}-x \tau_{i, b}\right)}$, and the total power consumption of mode $A$ is defined as $E_{\text {sum }}=2 E_{D}+$ $2 E_{C}+E_{R}$.

Proof Following that, based on Proposition 1, the CDF of SNDR at UE2, UE3 in AF scheme is given by

$$
F_{\gamma_{i}^{A F}}(x)=1-2 e^{-\omega_{i}^{A F}} \sqrt{\vartheta_{i}^{A F}} K_{1}\left(2 \sqrt{\vartheta_{i}^{A F}}\right)
$$

where $\vartheta_{i}^{A F}=\frac{\tau_{0} x}{\Omega_{X} \Omega_{Y}\left(\tau_{i, a}-x \tau_{i, b}\right)}, \omega_{i}^{A F}=\frac{x \tau_{i, c}}{\Omega_{X}\left(\tau_{i, a}-x \tau_{i, b}\right)}$. 
By evaluating the derivative of $F_{\gamma_{i}^{A F}}(x)$ with respect to $x$, and the PDF of $\gamma_{i}^{A F}$ can be written as

$$
\begin{aligned}
f_{\gamma_{i}^{A F}}(x) & =\frac{\partial}{\partial(x)} F_{\gamma_{i}^{A F}}(x) \\
& =M_{i}^{A F}+N_{i}^{A F},
\end{aligned}
$$

where $M_{i}^{A F}=\frac{2 \tau_{i, a} e^{-\omega_{i}^{A F}}{ }_{\vartheta_{i}^{A F}} K_{0}\left(2 \sqrt{\vartheta_{i}^{A F}}\right)}{x\left(\tau_{i, a}-x \tau_{i, b}\right)}, N_{i}^{A F}=$ $\frac{2 \tau_{i, a} \omega_{i}^{A F} e^{-\omega_{i}^{A F}} \sqrt{\vartheta_{i}^{A F}} K_{1}\left(2 \sqrt{\vartheta_{i}^{A F}}\right)}{x\left(\tau_{i, a}-x \tau_{i, b}\right)}$, and we derive the expression above by using the property of Bessel function in [[32], 8.486.18].

Thus, the closed-form expression for the average EE over Rayleigh fading channels can be given by

$$
\begin{aligned}
\mathbb{E}\left\{\log _{2}\left(1+\gamma_{i}^{A F}\right)\right\} & =\int_{\substack{x=0 \\
\tau_{i, a} / \tau_{i, b}}}^{\tau_{i, a} / \tau_{i, b}} f_{\gamma_{i}^{A F}(x) \log _{2}(1+x) d x} \\
& =\int_{x=0}^{A F}\left(M_{i}^{A F}+N_{i}^{A F}\right) \log _{2}(1+x) d x
\end{aligned}
$$

This proof is provided to prove Proposition 3.

Similar to Proposition 3, the analytical average EE and average SE in DF scheme in Mode A can be expressed as

$$
\begin{aligned}
e e_{i}^{D F} & =\mathbb{E}\left\{\frac{B \log _{2}\left(1+\gamma_{i}^{D F}\right)}{2 E_{\text {sum }}}\right\} \\
& =\frac{B}{2 E_{\text {sum }}} \int_{x=0}^{\psi_{i, a} / \psi_{i, b}}\left(M_{i}^{D F}+N_{i}^{D F}\right) \log _{2}(1+x) d x
\end{aligned}
$$

and

$$
\begin{aligned}
s e_{i}^{D F} & =\mathbb{E}\left\{\frac{B \log _{2}\left(1+\gamma_{i}^{D F}\right)}{2 B}\right\} \\
& =\frac{1}{2} \int_{x=0}^{\psi_{i, a} / \psi_{i, b}}\left(M_{i}^{D F}+N_{i}^{D F}\right) \log _{2}(1+x) d x
\end{aligned},
$$

where $M_{i}^{D F}=\frac{2 \psi_{i, a} e^{-\omega_{i}^{A F} \vartheta_{i}^{A F} K_{0}\left(2 \sqrt{\vartheta_{i}^{D F}}\right)}}{x\left(\psi_{i, a}-x \psi_{i, b}\right)}, N_{i}^{D F}=$ $\frac{2(1-\beta) \omega_{i}^{D F} e^{-\omega_{i}^{D F}} \sqrt{\vartheta_{i}^{D F}} K_{1}\left(2 \sqrt{\vartheta_{i}^{D F}}\right)}{x\left(\psi_{i, a}-x \psi_{i, b}\right)}$.

Meanwhile, when $\Gamma_{D}<1 / \kappa$, we derive the expression for both average EE and average SE in P2P communication with the transmission power and the circuit power, $P_{\text {sum }}=$ $2 E_{D}+2 E_{C}$ as

$$
\begin{aligned}
e e_{3}^{P P} & =2 \times \mathbb{E}\left\{\frac{B \log _{2}\left(1+\gamma_{3}^{P P}\right)}{2 P_{\text {sum }}}\right\} \\
& =\frac{B}{P_{\text {sum }} \ln 2} \int_{x=0}^{1 / \kappa} e^{-\frac{\Omega_{0} x}{\Omega_{W} E_{D} L_{W}(1-\kappa x)}}(1+x)^{-1} d x
\end{aligned}
$$

$$
\begin{aligned}
s e_{3}^{P P} & =2 \times \mathbb{E}\left\{\frac{B \log _{2}\left(1+\gamma_{3}^{P P}\right)}{2 B}\right\} \\
& =\frac{1}{\ln 2} \int_{x=0}^{1 / \kappa} e^{-\frac{\Omega_{0} x}{\Omega_{W} E_{D} L_{W}(1-\kappa x)}}(1+x)^{-1} d x
\end{aligned}
$$

\subsection{Optimization problem}

Regarding the HTPS relaying protocol, we try to solve the optimization problem of time switching (TS) and power spitting (PS) ratios with the aim to maximizing the STP. Therefore, we have the expression as follows

$$
\max _{\alpha_{1}, \alpha_{2}, \beta}\left\{2 e^{-\omega_{i}^{j}} \sqrt{\vartheta_{i}^{j}} K_{1}\left(2 \sqrt{\vartheta_{i}^{j}}\right)\right\}
$$

where the expression is subject to $\alpha_{1}, \alpha_{2}, \beta \in(0,1]$, and $\omega_{i}^{j}, \vartheta_{i}^{j}$ are defined in Propositions 1 and 2, respectively, $i \in\{1,2\}, j \in\{A F, D F\}$.

Due to the fact that the expression (33) is a non-convex function and it is difficult to derive analytic solution of $\alpha$, $\beta$ for HTPS relaying protocol, the achievement of optimal system configuration parameters cannot be done, since exhaustive search is not effective, where multiple parameters need to be optimized. To overcome this complication, we take advantage of a genetic algorithm (GA)-based optimization algorithm to obtain the optimal values of TS and PS to maximize the STP.

Definition 1 The generation of a random population is what GA begins with, which is defined as a set of chromosomes consisting of a group of genes and it holds values for the optimization variables [33]. Thanks to the evaluation of chromosome against an objective function, the fitness of each one is determined. In order to provide simulations for the natural survival of the fittest process, only best chromosomes can exchange information (via crossover or mutation) to produce offspring chromosomes. If offspring solutions are more feasible solutions than weak population members, they are investigated and used for population evolution. The process is continued for a vast number of generations to find a best-fit (near-optimum) solution. Note that the performance of GAs is affected by primary parameters, including number of generations, population size, crossover rate, and mutation rate [34, 35].

We regard TS fraction, $\alpha$, and PS fraction, $\beta$ as genes, respectively; we create a chromosome by the combination of $\alpha$ and $\beta$. In order to obtain each chromosome's fitness, objective function in (33) is used. $H_{\text {max }}$ denotes the optimal solution of the $t$-th generation, and the predefined precision with constraint tolerance of GA is denoted 
by $\epsilon$. Therefore, we present some steps of the GA-based optimization Algorithm 1 as follows:

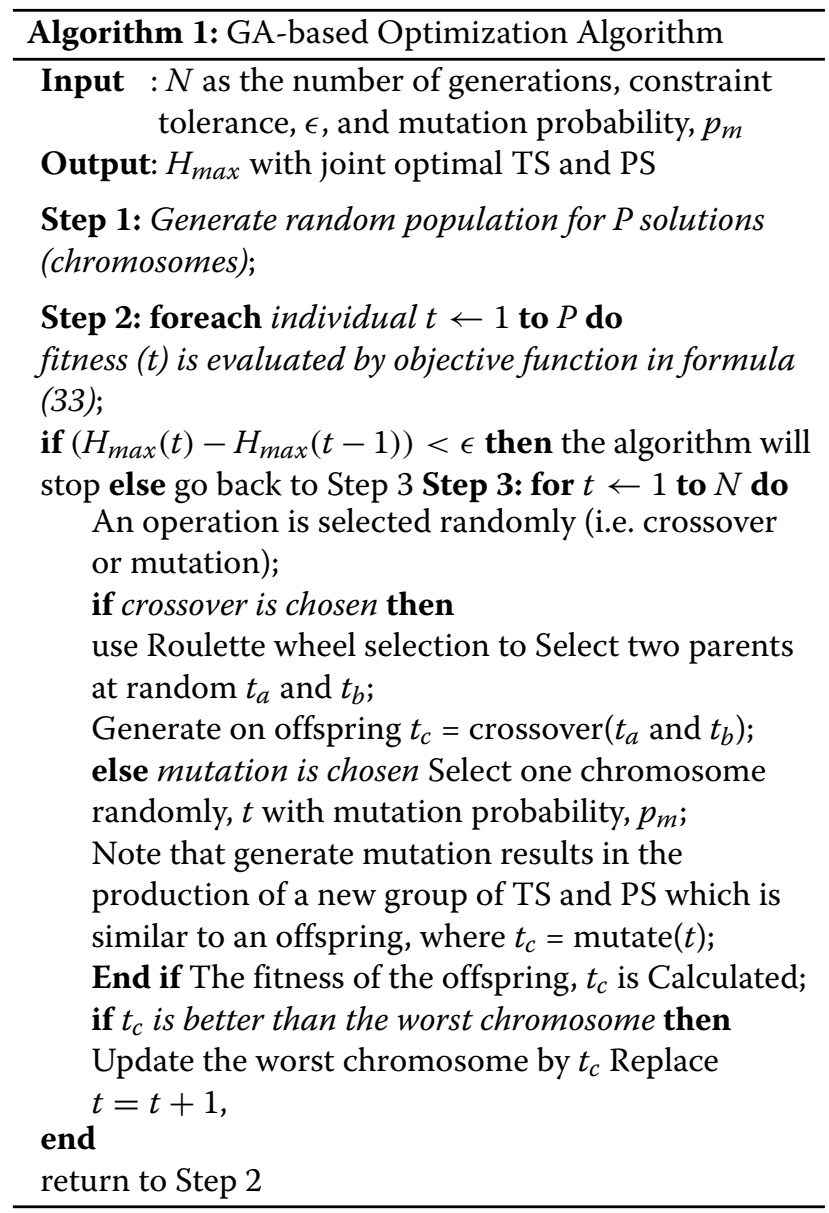

As a result, the use of a genetic algorithm (GA)-based optimization helps us achieve the joint optimal TS and PS ratios to guarantee the best STP.

\section{Numerical results and discussion}

In this section, we verify the accuracy of the analytical expressions through simulations and provide insight into the impact of HWIs on the STP and average EE and average SE in two transmission modes in the proposed HTPS relaying protocol, where comparisons between AF and DF in multi-hop D2D communication and P2P communication are provided. In addition, we also simulate the joint optimal values of TS and PS after the optimization problem is solved. In this part, primary parameters and default values are depicted in Table 2 which are used when UE1, R and UE2 are located at $(0,0),(0.5,0)$, and $(1,0)$ on the X-Y plane, respectively while UE3 is located at $(1,0.5)$. The simulation results are averaged over $10^{5}$ channel realizations.
Table 2 Main simulation parameters

\begin{tabular}{ll}
\hline Parameter & Values \\
\hline Circuit power at UEi, $E_{C}$ & $100 \mathrm{~W}$ \\
Channel bandwidth, $B$ & $10 \mathrm{Mhz}$ \\
$\begin{array}{l}\text { Path loss for all links in the system, } \\
P L_{k}, k \in[X, Y, Z, W]\end{array}$ & $\left(148+40 \log _{10}\left(r_{k}\right)\right)^{-1} \mathrm{~dB}$ \\
Thermal noise density, $\Omega_{0}$ & $-174 \mathrm{dBm} / \mathrm{Hz}$ \\
Energy conversion efficiency, $\eta$ & 1 \\
TS and PS ratios, $\alpha_{1}=\alpha_{2}=\beta$ & 0.1 \\
Power allocation, $\lambda$ & 0.7 \\
Hardware impairments level, $\kappa$ & 0.15 \\
SNDR threshold at UE2, $\Gamma_{D}$ & $3 \mathrm{~dB}$ \\
SNDR threshold at UE3, $\Gamma_{D}$ & $1 \mathrm{~dB}$ \\
\hline
\end{tabular}

\subsection{Successful transmission probability versus the maximum transmission, $E_{D}$ at UE2 and UE3}

As illustrated in Figs. 2 and 3, the successful transmission probability (STP) versus the maximum transmission, $E_{D}$ at UE2 and UE3 is considered, in which we compare AF and DF scheme using the HTPS protocol and TSR protocol under the impact HWIs. It is observed that the analytical results match well with simulation results. In particular, the increase in $E_{D}$ leads to better STP. It is clear that DF outperforms AF scheme, in which HTPS relaying protocol is better than TSR in terms of the STP. In addition, UE2 is much better than UE3 in the scenario without spectrum sharing, for example, when $E_{D}=10 \mathrm{~dB}$, the STP at UE2 is at approximately 0.55 and 0.72 for $\mathrm{AF}$ and $\mathrm{DF}$, respectively, while the STP at UE3 is at around 0.48 and 0.59 for $\mathrm{AF}$ and $\mathrm{DF}$, respectively.

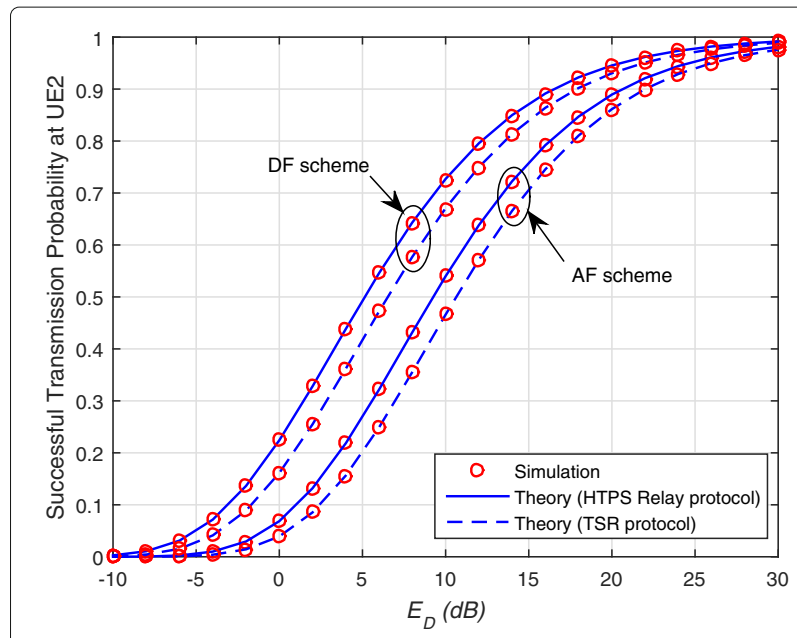

Fig. 2 STP versus $E_{D}(\mathrm{~dB})$ at UE2 


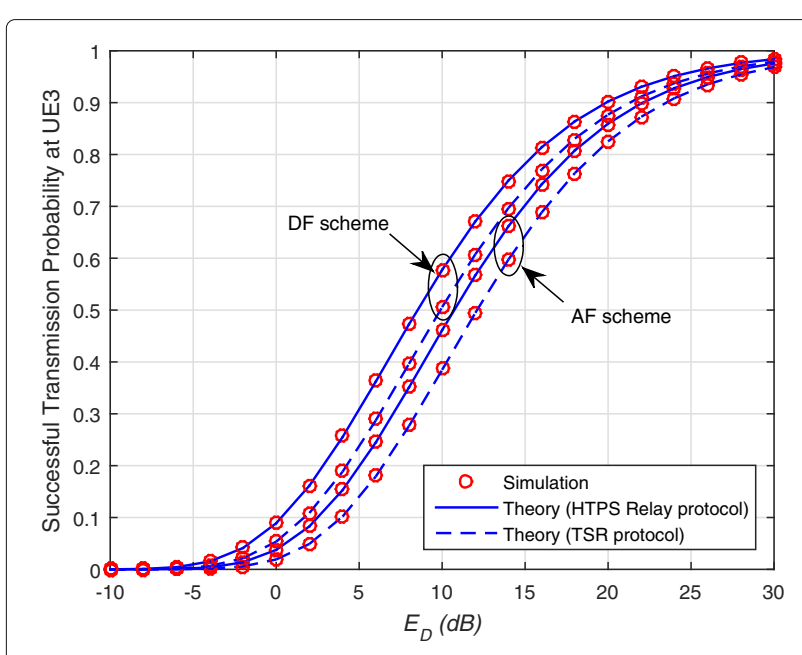

Fig. 3 STP versus $E_{D}(\mathrm{~dB})$ at UE3

\subsection{Successful transmission probability versus hardware impairment levels, $\kappa$ at UE2 and UE3}

Figures 4 and 5 illustrate the STP with levels of HWIs for $\mathrm{AF}$ and DF transmission scheme. The impact of HWIs on the evaluated STP is obvious, since some HWIs, i.e., transmit RF and receiver RF affect wireless communication systems. In particular, as $\kappa$ increases, it leads to the decrease in the STP, in which the STP in AF scheme drops remarkably when $E_{D}=5 \mathrm{~dB}$.

\subsection{Average energy efficiency and average spectral efficiency at UE2 and UE3 versus $E_{D}$}

In Figs. 6 and 7, we depict average energy efficiency (EE) and average spectral efficiency (SE) at UE2 as a function of $E_{D}(\mathrm{~W})$. It is evident that average EE increases as $E_{D}$ rises in the first half of the given period before declining steadily when $E_{D}$ is at around 0.2 , since the maximum

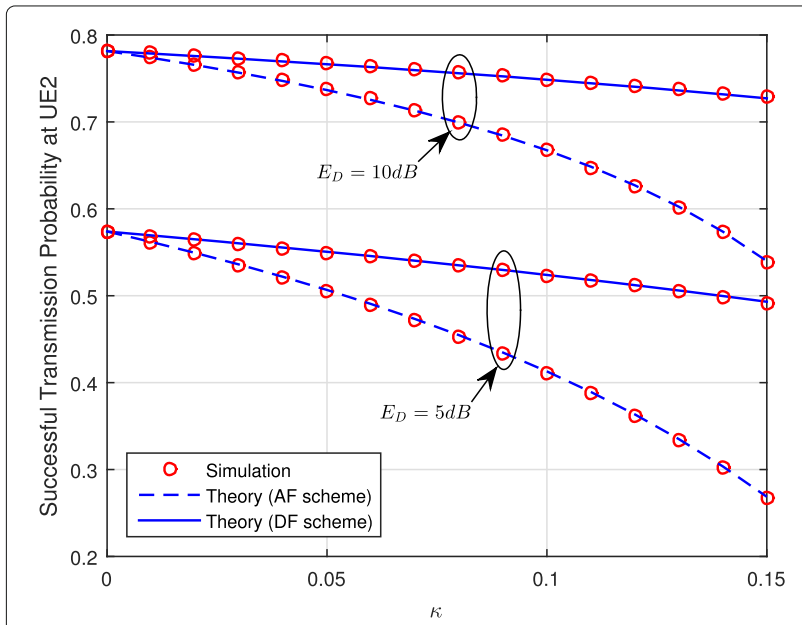

Fig. $4 \mathrm{STP}$ versus HWIs level, $\kappa$ with $E_{D}=10 \mathrm{~dB}$ and $E_{D}=5 \mathrm{~dB}$ at UE2

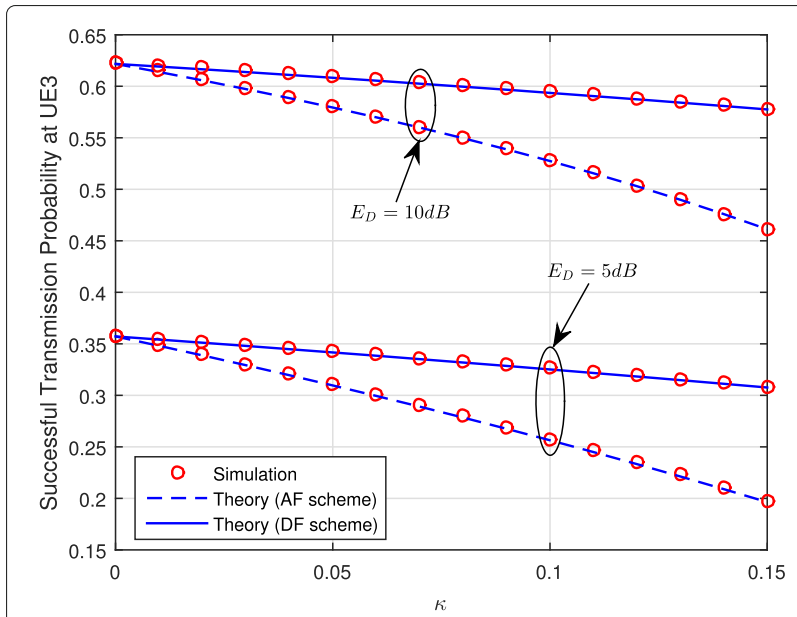

Fig. 5 STP versus HWIs level, $\kappa$ with $E_{D}=10 \mathrm{~dB}$ or $E_{D}=5 \mathrm{~dB}$ at UE3

transmission power, $E_{D}$ rises, which leads to the increase in $E_{\text {sum }}$ while the average SE is linear. Particularly, in Fig. 6, with two different levels of HWIs, $\kappa=0.15, \kappa=0.3$, the average EE is significantly affected, i.e, when $\kappa=0.3$, the average EE falls dramatically. Meanwhile, Fig. 7 shows the locations of $\mathrm{R}$ in two cases, including case 1 : $\mathrm{R}$ is located at $(0.5,0)$ and case $2: \mathrm{R}$ is located at $(0.3,0)$. As a result, the figure for case 2 is more remarkable, since the distance between UE2 and R is close, which accordingly leads to better SE. Furthermore, the similar trends at UE3 are presented in Figs. 8 and 9, it is evident that average EE falls as $E_{D}$ rises while the average $S E$ increase gradually in the given period, in which the performance gap between case 1 and case 2 is clear when the nearer the communication between R and UE3 is, the better SE is achieved. It is worth noting that the increase in $\lambda$ helps the primary node, UE2 achieves better EE and SE compared to UE3.

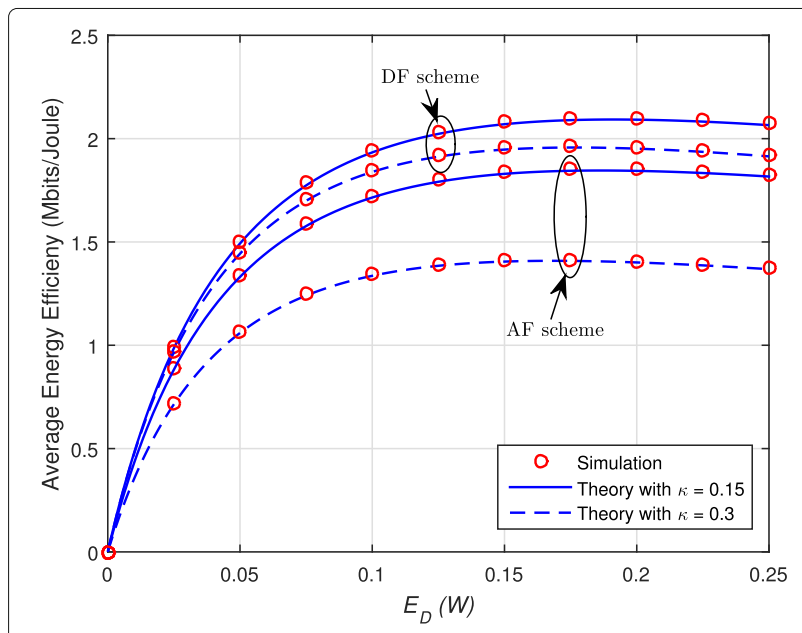

Fig. 6 Average EE versus $E_{D}(W)$ at UE2 


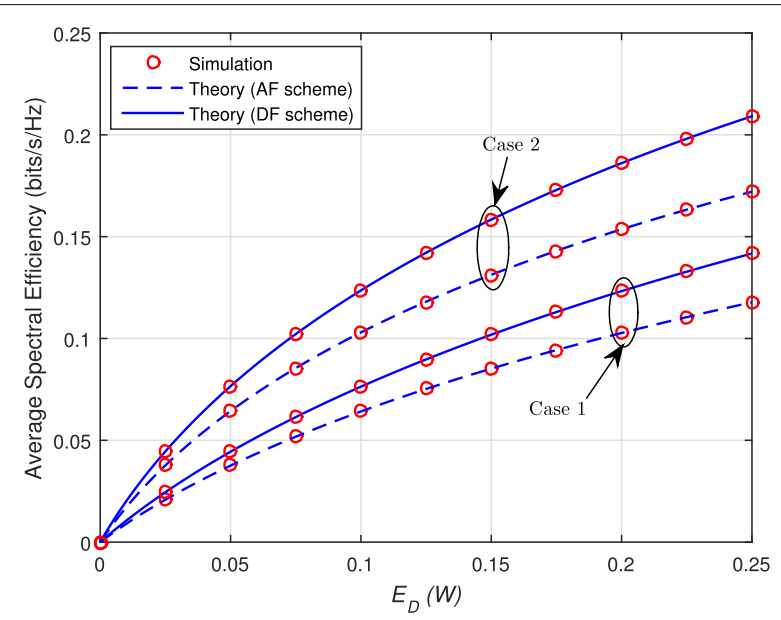

Fig. 7 Average $S E$ versus $E_{D}(W)$ in case 1 and case 2 at UE2

\subsection{Average energy efficiency and average spectral efficiency versus $E_{D}$ in the P2P communication}

The average EE and average SE as functions of $E_{D}(\mathrm{~W})$ in P2P communication are depicted in Fig. 10 in case the distance between UE2 and UE3 is $5 \mathrm{~km}$, while in Fig. 11, we compare two cases of the distance between the two nodes, including case 1 is $5 \mathrm{~km}$ and case 2 is $10 \mathrm{~km}$, respectively. The EE increases with SE at first, and after reaching a peak at approximately 87 and 68 when $\kappa=0.15$ and $\kappa=0.3$, respectively, it drops when average $\mathrm{SE}$ increases. Note that $\kappa=0.15$ means that SE suffers less from HWIs, so it goes up rapidly compared to the scenario when $\kappa=0.3$.

\subsection{Time switching and power splitting ratios under $\mathrm{HWis}$ at UE2 and UE3}

In Figs. 12 and 13, we provide visual results under different TS and PS ratios for UE2 and UE3 under the impact of HWIs. As illustrated in the two figures, the STP in case

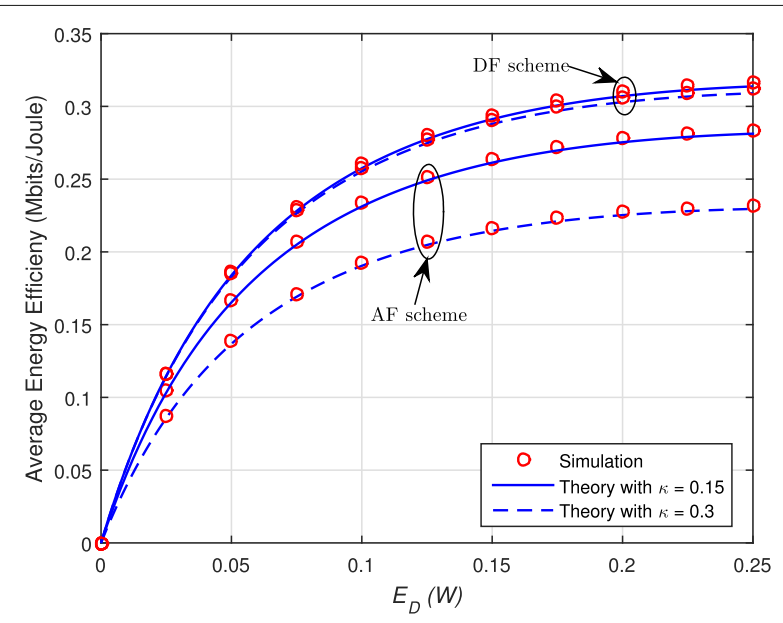

Fig. 8 Average $E E$ versus $E_{D}(W)$ at UE3

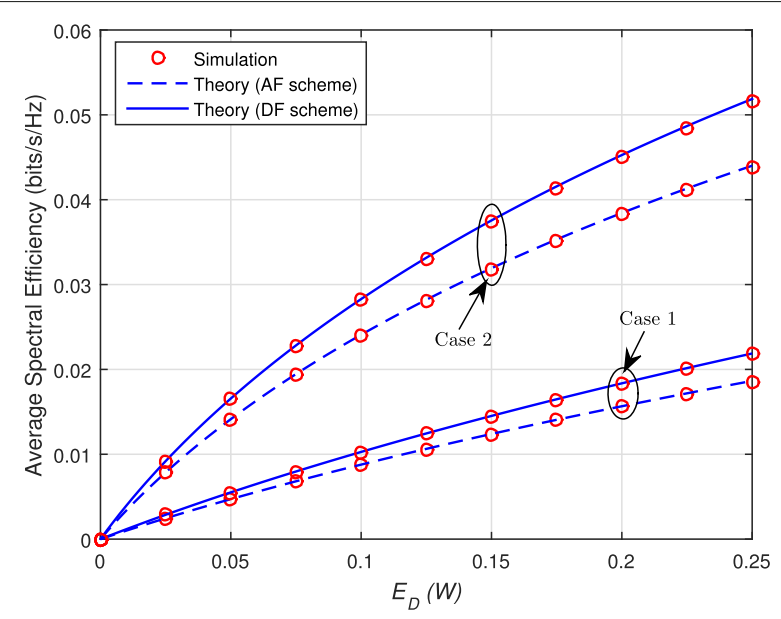

Fig. 9 Average $S E$ versus $E_{D}(W)$ in case 1 and case 2 at UE3

of the hybrid HTPS relaying protocol achieves its optimal values when the TS and PS fraction pair ranges from 0 to $0.7,0$ to 0.4 , respectively, with parameters, i.e., $E_{D}=10 \mathrm{~dB}$ and $\kappa=0.15$.

\subsection{The joint optimal value of $\alpha$ and $\beta$ versus $E_{D}$ at UE2 and UE3}

Figures 14 and 15 present the results of optimization problem achieved in Section 3.4 with number of generations, $N=100$, constraint tolerance, $\epsilon=10^{-5}$, mutation probability, $p_{m}=0.05$, respectively. The joint optimization results are compared with fixed values of TS and PS fraction with $\alpha_{1}=\alpha_{2}=\beta=0.1$ at UE2 and UE3 in AF and DF scheme. It can be observed that all curves are going up as $E_{D}$ increases because the higher $E_{D}$ means larger SDNR, which increases the STP. Furthermore, the performance gain of the joint optimized TS and PS achieves

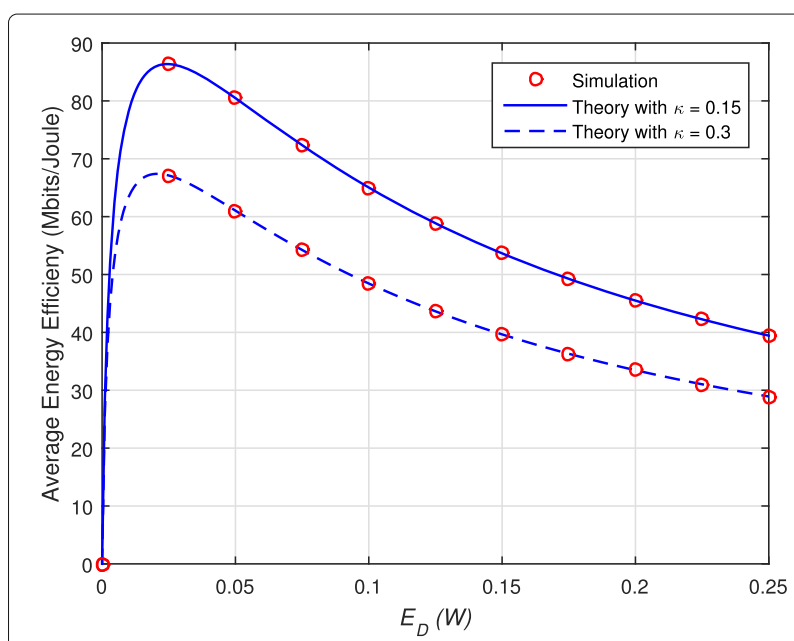

Fig. 10 Average $E E$ versus $E_{D}(W)$ in $P 2 P$ communication under the impact of HWIs 


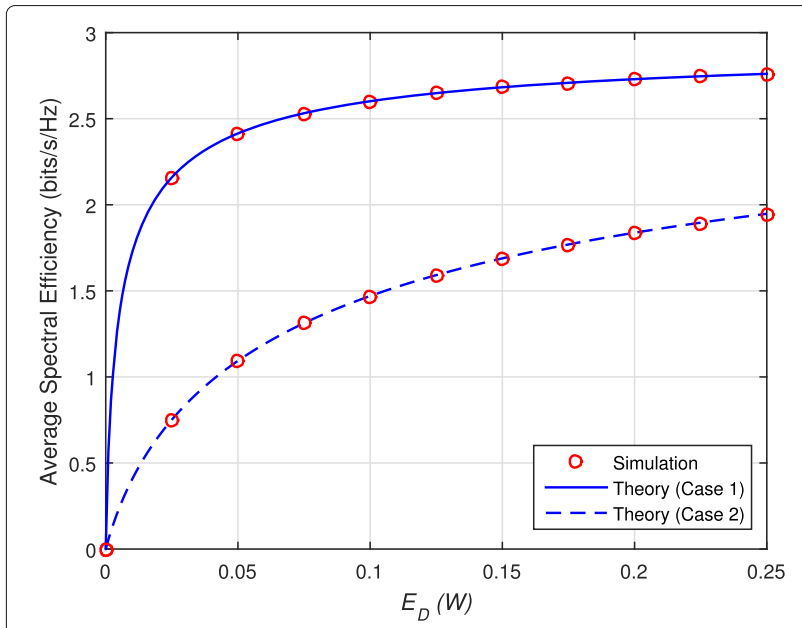

Fig. 11 Average $S E$ versus $E_{D}(W)$ in P2P communication under the impact of HWls

maximum value when $E_{D}$ is at approximately $25 \mathrm{~dB}$ and the performance gap is more obvious in DF scheme.

\section{Conclusions}

In this paper, we addressed the impact of HWIs on the cognitive energy harvesting-based D2D communication underlaying cellular network consisting of two communication types, i.e., multi-hop D2D and P2P. Closed-form expressions for the successful transmission probability, average EE and SE were derived, and we also solved the optimization problem with respect to TS and PS ratios using a genetic algorithm (GA)-based optimization algorithm. Simulation results were given to verify the comparisons between $\mathrm{AF}$ and $\mathrm{DF}$ transmission schemes, where DF outperforms AF in terms of every parameter. Most importantly, although all nodes suffered from HWIs, the STP was still guaranteed.

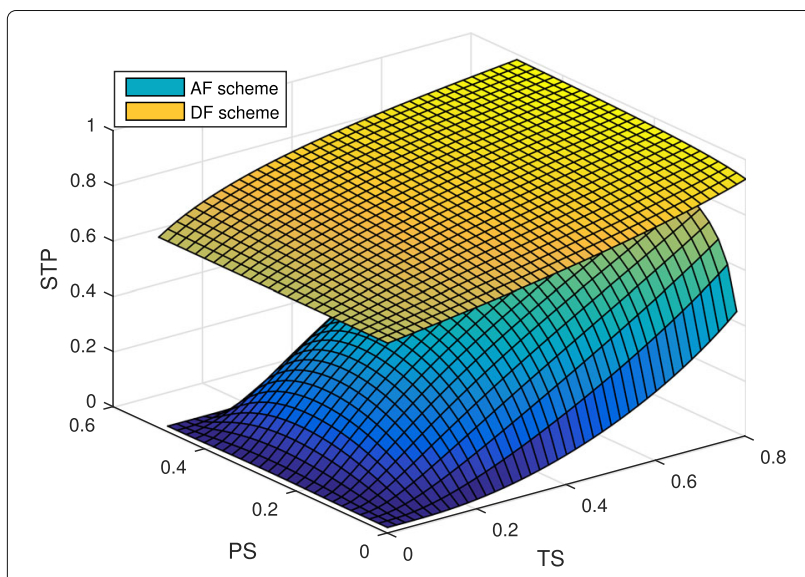

Fig. 12 STP versus TS and PS at UE2
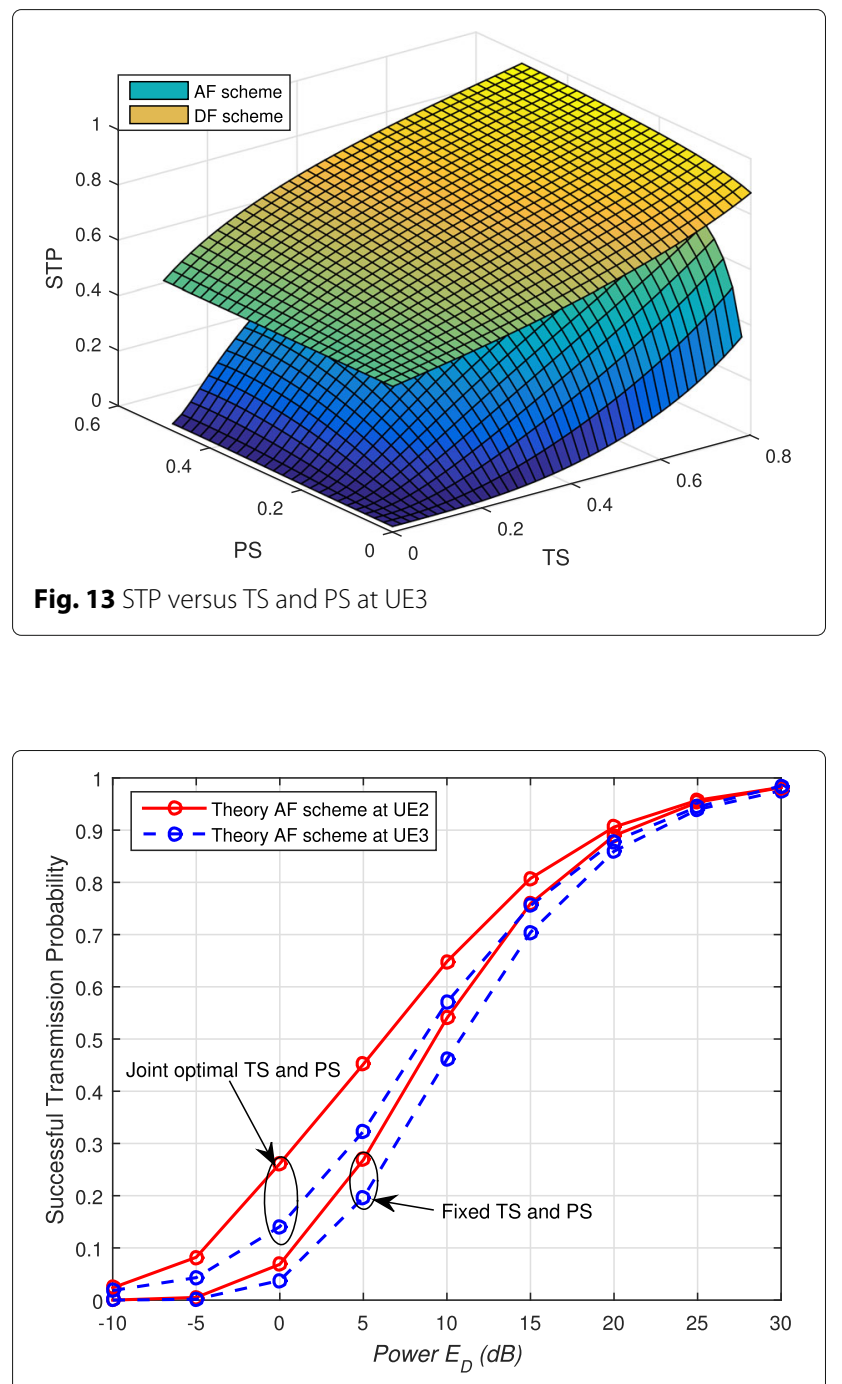

Fig. $14 \mathrm{STP}$ versus $E_{D}(\mathrm{~dB})$ in $A F$ scheme

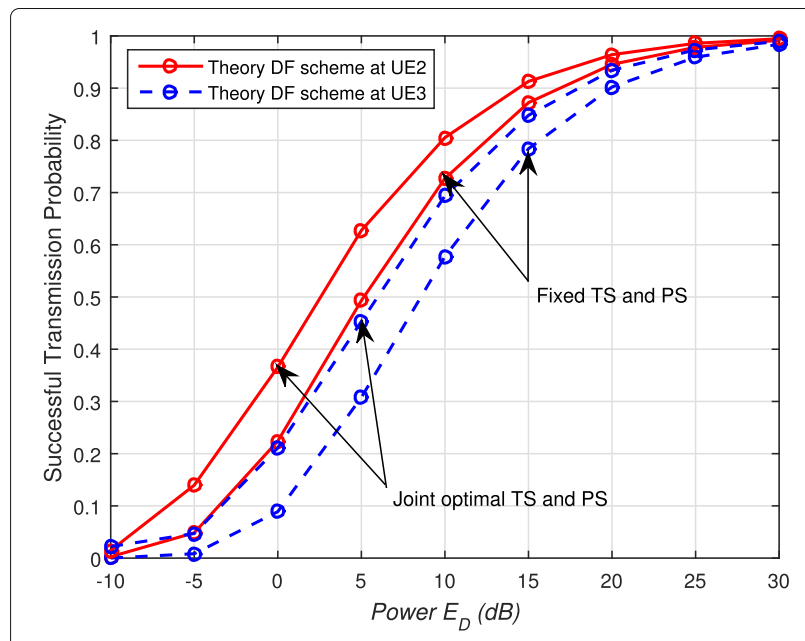

Fig. $15 S T P$ versus $E_{D}(d B)$ in DF scheme 


\section{Authors' contributions}

HSN contributed in the conception and design of the study; he also carried out the simulation and revised the manuscript. HSN helped to perform the analysis with constructive discussions. Meanwhile, TSN was responsible for producing simulation analysis and writing the manuscript carefully. Furthermore, MV went through and approved the final manuscript. All authors read and approved the final manuscript.

\section{Competing interests}

The authors declare that they have no competing interests.

\section{Publisher's Note}

Springer Nature remains neutral with regard to jurisdictional claims in published maps and institutional affiliations.

\section{Author details}

${ }^{1}$ Wireless Communications Research Group, Faculty of Electrical \& Electronics Engineering, Ton Duc Thang University, Ho Chi Minh City, Vietnam. ${ }^{2}$ Binh Duong University, Thu Dau Mot City, Binh Duong Province, Vietnam. ${ }^{3}$ VSB-Technical University of Ostrava, 17. listopadu 15/2172, 70833 Ostrava, Czech Republic.

\section{Received: 14 June 2017 Accepted: 16 November 2017} Published online: 08 December 2017

\section{References}

1. VD Nguyen, TQ Duong, HD Tuan, OS Shin, H Vincent, Poor, Spectral and Energy Efficiencies in full-duplex wireless information and power transfer. IEEE Trans. Commun. 65(6), 2220-2233(2017). doi:10.1109/TCOMM.2017.2665488

2. $Y$ Ren, $T L v, H$ Gao, $Y$ Li, Secure wireless information and power transfer in heterogeneous networks. IEEE Access. 5, 4967-4979 (2017). doi:10.1109/ACCESS.2017.2682277

3. $\mathrm{X} \mathrm{Di}$, K Xiong, P Fan, $\mathrm{HC}$ Yang, Simultaneous wireless information and power transfer in cooperative relay networks with rateless codes. IEEE Trans. Veh. Technol. 66(4), 2981-2996 (2016). doi:10.1109/TVT.2016.2588441

4. AA Nasir, X Zhou, S Durrani, RA Kennedy, Relaying protocols for wireless energy harvesting and information processing. IEEE Trans. Wirel. Commun. 12(7), 3622-3636 (2013). doi:10.1109/TWC.2013.062413.122042

5. Z Wang, Z Chen, B Xia, L Luo, J Zhou, Cognitive relay networks with energy harvesting and information transfer: design, analysis and optimization. IEEE Trans. Wirel. Commun. 15(4), 2562-2576 (2016). doi:10.1109/TWC.2015.2504581

6. HS Nguyen, DT Do, M Voznak, Two-way relaying networks in green communications for $5 \mathrm{G}$ : optimal throughput and tradeoff between relay distance on power splitting-based and time switching-based relaying SWIPT. AEUE. Int. J. Electron. Commun. 70(3), 1637-1644 (2016). doi:10.1016/j.aeue.2016.10.002

7. KXiong, P Fan, C Zhang, KB Letaief, Wireless information and energy transfer for two-hop non-regenerative MIMO-OFDM relay networks. IEEE J. Sel. Areas Commun. 33(8), 1595-1611 (2015). doi:10.1109/JSAC.2015.2391931

8. J Gao, On the successful transmission probability of cooperative cognitive radio ad hoc networks. Ad Hoc Netw. 58, 99-104 (2017). doi:10.1016/j.adhoc.2016.10.012

9. RHY Louie, Y Li, B Vucetic, Practical physical layer network coding for twoway relay channels: performance analysis and comparison. IEEE Trans. Wirel. Commun. 9(2), 764-777 (2010). doi:10.1109/TWC.2010.02.090314

10. KXiong, C Chen, G Qu, P Fan, KB Letaief, Group cooperation with optimal resource allocation in wireless powered communication networks. IEEE Trans. Wirel. Commun. 16(6), 1536-1276 (2017). doi:10.1109/TWC.2017.2689011

11. L Liu, R Zhang, KC Chua, Wireless information and power transfer: a dynamic power splitting approach. IEEE Trans. Commun. 61(9), 3990-4001 (2013). doi:10.1109/TCOMM.2013.071813.130105

12. KXiong, P Fan, Y Lu, KB Letaief, Energy efficiency with proportional rate fairness in multirelay OFDM networks. IEEE J. Sel. Areas Commun. 34(5), 0733-8716 (2016). doi:10.1109/JSAC.2016.2545479

13. A Singh, MR Bhatnagar, RK Mallik, Secrecy outage of a simultaneous wireless information and power transfer cognitive radio system. IEEE Commun. Lett. 5(3), 288-291 (2016). doi:10.1109/LWC.2016.2544828

14. K An, J Ouyang, M Lin, T Liang, Outage analysis of multi-antenna cognitive hybrid satellite-terrestrial relay networks with beamforming. IEEE Wirel. Commun. Lett. 19(7), 1157-1160 (2015). doi:10.1109/LCOMM.2015.2428256
15. R Manna, RHY Louie, Y Li, B Vuceticg, Cooperative spectrum sharing in cognitive radio networks with multiple antennas. IEEE Trans. Signal Process. 59(11), 5509-5522 (2011). doi:10.1109/TSP.2011.2163068

16. M Karaca, K Khalil, E Ekici, O Ercetin, Optimal scheduling and power allocation in cooperate-to-join cognitive radio networks. IEEE/ACM Trans. Netw. 21(6), 1708-1721 (2013). doi:10.1109/TNET.2012.2230187

17. X Bao, P Martins, T Song, L Shen, Capacity of hybrid cognitive network with outage constraints. IET Commun. 5(18), 2712-2729 (2011). doi:10.1049/iet-com.2011.0236

18. Feasibility Study for Proximity Service (ProSe). 3GPP TR 22.803 V12.2.0, (2013)

19. K Doppler, M Rinne, C Wijting, C Ribeiro, K Hugl, Device-to-device communications as an underlay to LTE-advanced networks. IEEE Commun. Mag. 47(12), 42-49 (2010). doi:10.1109/WCNC.2010.5506248

20. ST Su, BY Huang, CY Wang, CW Yeh, HY Wei, Protocol design and game theoretic solutions for device-to-device radio resource allocation. IEEE Trans. Veh. Technol. 66(5), 4271-4286 (2017). doi:10.1109/TVT.2016.2602658

21. Z Xia, J Yan, Y Liu, Cooperative content delivery in multicast multihop device-to-device networks. IEEE Access. 5, 6314-6324 (2017). doi:10.1109/ACCESS.2017.2672996

22. X Chai, T Liu, CXing, H Xiao, Z Zhang, Throughput improvement in cellular networks via full-duplex based device-to-device communications. IEEE Access. 4, 7645-7657 (2017). doi:10.1109/ACCESS.2016.2619379

23. E Bjornson, J Hoydis, M Kountouris, M Debbah, Massive MIMO systems with non-ideal hardware: Energy efficiency, estimation, and capacity limits. IEEE Trans. Inf. Theory. 60(11), 7112-7139 (2014). doi:10.1109/TIT.2014.2354403

24. XXia, D Zhang, KXu, W Ma, Y Xu, Hardware impairments aware transceiver for full-duplex massive MIMO relaying. IEEE Trans. Signal Process. 63(24), 6565-6580 (2015). doi:10.1109/TSP.2015.2469635

25. NT Do, DBD Costa, B An, Performance analysis of multirelay RF energy harvesting cooperative networks with hardware impairments. IET Commun. 10(18), 2551-2558 (2016). doi:10.1049/iet-com.2016.0392

26. TP Huynh, HS Nguyen, DT Do, M Voznak, in Proc.of International Conference on Electronics, Information, and Communications, (ICEIC). Impact of hardware impairments in AF relaying network for WIPT: TSR and performance analysis, (2016), pp. 1-4. doi:10.1109/ELINFOCOM.2016. 7562967

27. HS Nguyen, H Nguyen, SD Sau, M Voznak, TP Huynh, in Proc.of International Conference on Electronics, Information, and Communications, (ICEIC). Impact of hardware impairments for power splitting relay with wireless information and EH, (2016), pp. 1-4. doi:10.1109/ELINFOCOM.2016.7562941

28. TN Nguyen, PT Tran, HG Hoang, HS Nguyen, M Voznak, in Proc.of International Conference on Advances in Information and Communication Technology, (ICACT). On the performance of decode-and-forward half-duplex relaying with time switching based energy harvesting in the condition of hardware impairment, (2017), pp. 421-430. doi:10.1007/978-3-319-49073-1_46

29. L Wei, RQ Hu, Y Qian, G Wu, Energy efficiency and spectrum efficiency of multihop device-to-device communications underlaying cellular networks. IEEE Trans. Veh. Technol. 65(1), 367-380 (2016). doi:10.1109/TVT.2015.2389823

30. T Schenk, RF Imperfections in high-rate wireless systems: impact and digital compensation. (Springer Publishing, 2010). doi:1 0.1007/978-1-4020-6903-1

31. M Matthaiou, A Papadogiannis, E Bjornson, Debbah M, Two-way relaying under the presence of relay transceiver hardware impairments. IEEE Commun. Lett. 17(6), 1136-1139 (2013). doi:10.1109/LCOMM.2013.042313.130191

32. IS Gradshteyn, IM Ryzhik, Table of integrals, series, and products, 4th edn. (Academic Press Inc., New York, 1980)

33. DE Goldberg, Genetic algorithms in search, optimization and machine learning. (Addison-Wesley Longman Publishing Co., Inc., Boston, 1989)

34. KF Man, KS Tang, S Kwong, Genetic algorithms: concepts and applications. IEEE Trans. Ind. Electron. 43(5), 519-534 (1996). doi:10.1109/41.538609

35. G Du, K Xiong, Y Zhang, Z Qiu, Outage analysis and optimization for time switching-based two-way relaying with energy harvesting relay node. KSII Trans. Internet Inf Syst. 9(2), 1-19 (2014). doi:10.3837/tiis.2015.02.004 